\title{
Shrinkage Model Selection for Portfolio Optimization on Vietnam Stock Market*
}

\author{
Nhat NGUYEN 1 , Trung NGUYEN ${ }^{2}$, Tuan TRAN ${ }^{3}$, An MAI $^{4}$
}

Received: July 03, 2020 Revised: July 25, 2020 Accepted: August 10, 2020

\begin{abstract}
This paper provides the practical application of a linear shrinkage framework on Vietnam stock market. The cumulative data points observed in this analysis are 468 weeks from January 2011 to December 2019. All the companies listed on Ho Chi Minh City Stock Exchange (HOSE), except the companies under two years period from Initial Public Offering (IPO), are considered. The cumulative number of stocks picked is therefore 350 companies. The VNINDEX, which is the Vietnam Stock Index, is used as a reference index for shrinking to a single-index model. The empirical results show that the shrinkage of covariance matrix for portfolio optimization gives the promising results for the investors on Vietnam stock market. The shrinkage method helps the investors to produce the optimal portfolio in the sense of having higher profit with lower levels of risk compared to the portfolio of the traditional SCM method. Moreover, the portfolio turnover of shrinkage method is always kept at low magnitudes, and this makes the shrinkage portfolios save much transaction costs and reduce the liquidity risks in the trading process. In addition, the ability of shrinkage method in making profit is once again confirmed by the Alpha coefficient that achieves a high positive value.
\end{abstract}

Keywords: Shrinkage Estimator, Single-Index-Model, Constant Correlation Model, Identity Matrix

JEL Classification Code: C51, C55, G11

\section{Introduction}

Modern Portfolio Theory (MPT) has been playing a significant role in constructing investment portfolios for

\footnotetext{
*Acknowledgements:

The study was supported by The Youth Incubator for Science and Technology Program, managed by Youth Development Science and Technology Center - Ho Chi Minh Communist Youth Union and Department of Science and Technology of Ho Chi Minh City, the contract number is " $14 / 2019$ / HĐ-KHCN-VU" ".

${ }^{1}$ First Author and Corresponding Author. Lecturer, Faculty of Banking, Banking University of Ho Chi Minh City, Vietnam [Postal Address: 36 Ton That Dam Street, Nguyen Thai Binh Ward, District 1, Ho Chi Minh City, 700000, Vietnam] Email: nhatnm@buh.edu.vn

${ }^{2}$ Associate Professor, Faculty of Banking, Banking University of Ho Chi Minh City, Vietnam. Email: trungnd@buh.edu.vn

3Junior Researcher, ICT Department, John von Neumann Institute, Vietnam National University. Email: tuan.tran@jvn.edu.vn

${ }^{4}$ Senior Researcher, ICT Department, John von Neumann Institute, Vietnam National University. Email: an.mai@jvn.edu.vn

(c) Copyright: The Author(s)

This is an Open Access article distributed under the terms of the Creative Commons Attribution Non-Commercial License (https://creativecommons.org/licenses/by-nc/4.0/) which permits unrestricted non-commercial use, distribution, and reproduction in any medium, provided the original work is properly cited.
}

over 65 years, since its first introduction in 'Portfolio Selection' article in Journal of Finance. The framework of MPT is to attain the highest return possible for a certain level of risk through structuring the optimal weights of various assets (Iyiola, 2012). Although it has been applied in real-life investments, the assumptions have been greatly challenged, especially in the volatile scenario. The main reasons come from the two main inputs of MPT, which are the mean of asset return and the corresponding covariance matrix.

To implement this MPT framework in practice, investors must approximate the mean asset return and the covariance matrix of all assets. At this point, sample mean as well as covariance matrix approaches are usually employed. However, they are unreliable in multiple cases due to estimation errors, especially in the high-dimensional portfolios, which makes the weights of assets in the portfolio fluctuate continuously over time and the transaction costs higher. Therefore, the theory is difficult to use in reality by the portfolio managers. Moreover, many renowned empirical studies have shown that these portfolios underperformed in term of mean and variance metrics during the out-of-sample period (Michaud, 1989). 
Estimating a high-dimensional covariance matrix for portfolio optimization is a longstanding challenge. Getting large dimensionality for covariance matrix means that it is more frequently to get unexpected and ungovernable errors in some sorts of computational steps, and the sample data might not be sufficient for the true covariance matrix estimation. To address this issue, there have been many approaches proposed in the literature, and among them, Ledoit and Wolf (2003a, 2003b, 2004) proposed to select the optimal portfolios by using the shrinkage estimation. This method is a combination between a rough sample covariance matrix and a high-structured target matrix to achieve the balance between bias and variance. The balance can be customized, which is the trade-off between bias and estimation errors recognized by shrinkage coefficients. The shrinkage technique clearly shows theoretically and empirically attractive approach to a high-dimensional portfolio's covariance estimation problem since it ensures a well-defined covariance matrix is achieved.

To our knowledge, there is a lack of in-depth research and application of shrinkage methodologies to Vietnam stock market. Therefore, we discuss in this paper the practical application of Linear Shrinkage framework of Ledoit and Wolf on Vietnam stock market as a pilot research. The experimental studies show promising results, which not only encourage the investors to focus more and more on the approach of portfolio selection based shrinkage frameworks, but also support them to select the suitable the target matrix on shrinkage method.

\section{Literature Review}

The shrinkage technique's success comes from selecting an appropriate shrinkage target, but investors do not find this very easy. The shrinkage method was first mentioned by Ledoit and Wolf (2003a) and used a single-index model (SSIM), which is the target matrix in the shrinkage method. Results show that the shrinkage towards single-index model results is superior to the conventional sample covariance matrix (SCM) in terms of both return and level of risk tested on out-of-sample data. Ledoit and Wolf (2003b) continued to use the shrinkage method to select a portfolio on the US stock market, but with another target matrix, the constant correlation model (CCM). The results show that the shrinkage, which shrinks towards constant correlation model (SCCM) does somewhat better than the shrinkage towards single-index model (SSIM) for $\mathrm{N} \leq 100$ and is somewhat worse for $N \geq 225$. Meanwhile, SCCM also has better results than PC-5 (the estimator relied on the first five principal components) for $\mathrm{N} \leq 50$ and is comparable to PC-5 for $\mathrm{N} \geq 100$. Ledoit and Wolf (2004) introduced another shrinkage target matrix, which is an identity matrix. The results showed that the shrinkage to identity matrix
(STIM) outperformed the traditional covariance matrix, as the size of the portfolio increased, the more pronounced was the difference. Due to the nature of shrinkage methods, which often provides strong experimental results, this approach becomes a benchmark for portfolio selection in recent years.

Liu (2014) estimated the covariance matrix by applying the weighted average of different shrinkage target matrices, instead of using a single shrinkage target matrix as Ledoit and Wolf method. Next, by inheriting the potentials and development of Random Matrix Theory, Ledoit and Wolf extended their pilot works (Ledoit \& Wolf, 2017a, 2017b) by using a nonlinear transformation applied for the eigenvalues considering solely the sample data. Also, coefficient asymptotically leads to the maximization of the out-of-sample expected utility. Then, they performed both numerical and empirical investigation where the out-of-sample behavior of the obtained estimator is analyzed and it shows remarkable improvements over the simple diversification, and its robustness is expressed to the deviations from normality. As a matter of fact, DeMiguel et al. (2013) provided an important review paper of shrinkage frameworks and their practical application especially for asset optimization, and then they also discussed on a new category of shrinkage-based techniques for the means of return and the corresponding covariance matrix, as well as, the weights in the asset. As a slight enhancement for this research approach, the work of Candelon et al. (2012) presented such a kind of double shrinkage adaptation to improve the general stability of the estimation on even small sample sizes covariance matrices via taking into account a ridge regression approach to shrink the all the weights towards the equally-weighted asset.

\section{Theoretical Framework}

\subsection{Linear Shrinkage Estimator of Covariance Matrix}

Linear shrinkage estimation is the mix of the covariance matrix estimated by sample and the shrinkage target matrix through a weight is called the shrinkage intensity $-\sigma$, which is shown as follows: $\sum_{\text {Shrinkage }}=(1-\sigma) S_{S C M}+\sigma \sum_{\text {target }}(0 \leq \sigma \leq 1)$. The shrinkage target is usually a matrix that has high structure. The shrinkage can take advantage of both the sample covariance matrix (SCM) and the target matrix while eliminating their limitations. By determining the most fitting weights between the two-covariance matrices that are calculated from the SCM and the shrinkage target, the estimated covariance matrix can be similar to the true covariance matrix. Thus, there are three elements to be defined in the linear shrinkage method: the sample covariance matrix, the target matrix, and the shrinkage intensity. 


\subsubsection{Sample Covariance Matrix (SCM)}

Assuming that $r_{i, t}, r_{j, t}$ are the historical returns of assets $\mathrm{i}$ and $\mathrm{j}$ at the time period t. The historical average returns of asset i $\left(\bar{r}_{i}\right)$ and asset $\mathrm{j}\left(\bar{r}_{j}\right)$ in the period $[1, \mathrm{~T}]$ will be calculated as follows:

$$
\overline{r_{i}}=\frac{1}{T} \sum_{t=1}^{T} r_{i, t} \quad \text { and } \quad \bar{r}_{j}=\frac{1}{T} \sum_{t=1}^{T} r_{j, t}
$$

The equation that is used to calculate the sample covariance between any two assets $i, j$ is:

$$
\operatorname{Cov}\left(r_{i}, r_{j}\right)=\frac{1}{T-1} \sum_{t=1}^{T}\left(r_{i, t}-\bar{r}_{i}\right)\left(r_{j, t}-\bar{r}_{j}\right):=\hat{\sigma}_{i j}
$$

From the equation (2), the sample covariance matrix $\left(\widehat{\Sigma_{S C M}}\right)$ that shows the relationship among $\mathrm{N}$ assets in the portfolio is identified as follows:

$$
\widehat{\Sigma_{S C M}}=\left[\begin{array}{cccc}
\hat{\sigma}_{11} & \hat{\sigma}_{12} & \ldots & \hat{\sigma}_{1 N} \\
\hat{\sigma}_{21} & \hat{\sigma}_{22} & \ldots & \hat{\sigma}_{2 N} \\
\vdots & \vdots & \ddots & \vdots \\
\hat{\sigma}_{N 1} & \hat{\sigma}_{N 2} & \ldots & \hat{\sigma}_{N N}
\end{array}\right]
$$

\subsubsection{Shrinkage Target Matrix}

There are three shrinkage target matrices that are studied in this paper, including single-index model (SIM), constant correlation model (CCM) and identity matrix (IM). The shrinkage target matrices will be identified as follows:

\section{Single-index model (SIM):}

This model assumes that the assets' returns are significantly influenced by the market return. Thus, the estimated return of an asset i $\left(\bar{r}_{i . t}\right)$ will be identified through a following regression model:

$$
\hat{r}_{i . t}=\alpha_{i}+\beta_{i} \hat{r}_{m}+\varepsilon_{i, t}
$$

where: $\hat{r}_{m}$ is the estimated market return, $\varepsilon_{i, t}$ is the random error, $\alpha_{i}$ and $\beta_{i}$ are coefficient calculated from the regression. Assumptions of this model are that $\varepsilon_{i, t}$ is independent; $\operatorname{Cov}\left[\varepsilon_{i}, \varepsilon_{j}\right]$ is equal 0 and $\operatorname{Cov}\left[\hat{r}_{m}, \varepsilon_{i}\right]=0$. Moreover, the error $\left(\varepsilon_{i, t}\right)$ will follow a normal distribution with Var $\left[\varepsilon_{i}\right]=\sigma_{\varepsilon_{i}}^{2}$ and $\mathrm{E}\left[\varepsilon_{i}\right]=0$.

The variance and covariance of estimated asset returns $i$, $\mathrm{j}$ are measured by these following formulas:

$$
\operatorname{Var}\left[\hat{r}_{i}\right]=\beta_{i}^{2} \hat{\sigma}_{i}^{2}+\sigma_{\varepsilon_{i}}^{2} \text { and } \operatorname{Cov}\left[\hat{r}_{i}, \hat{r}_{j}\right]=\beta_{i} \beta_{j} \hat{\sigma}_{m}^{2}
$$

The covariance matrix calculated by SIM is as follows:

$$
\hat{\Sigma}_{S I M}=\beta \beta^{T} \hat{\sigma}_{m}^{2}+\hat{\Sigma}_{\varepsilon}
$$

Where $\hat{\sigma}_{m}=\operatorname{Var}\left[\hat{r}_{m}\right]$ is the estimated market variance; $\hat{\Sigma}_{\varepsilon}$ is the matrix of regression's error with the shape of $\mathrm{N} x \mathrm{~N}$ diagonal matrix.

\section{Constant correlation model (CCM):}

The covariance matrix estimated from the CCM is based on the premise that all stock pairs in the portfolio have the same correlations and equal to the mean correlation. Therefore, the constant correlation matrix is calculated as follows:

First, the sample correlations between stocks i, $j$ is calculated by:

$$
r_{i j}=\frac{S_{i j}}{\sqrt{S_{i i} S_{j j}}}
$$

In which: $\mathrm{S}$ is the sample covariance matrix and $s_{i, j}$ is the element of the matrix $\mathrm{S}$.

Second, the average of sample correlations is calculated by the equation:

$$
\bar{r}=\frac{2}{(N-1) N} \sum_{i=1}^{N-1} \sum_{j=i+1}^{N} r_{i j}
$$

Finally, constant correlation matrix $(\mathrm{C})$ is defined as:

$$
C_{i i}=S_{i i} \text { and } C_{i j}=\bar{r} \sqrt{S_{i i} S_{i j}}
$$

\section{Identity matrix:}

Identity matrix (IM) is defined as a square matrix in which the elements of main diagonal are all one and the other elements of matrix are all zero. There is a major difference between shrinkage towards identity matrix and shrinkage towards other target matrices. The shrinkage towards other target matrices require a good selection of target matrices that should be depend on some known features of the true covariance matrix for applying in the real life. For example, Ledoit and Wolf exploited the first known feature that stock returns have a factor-model structure to calculate covariance matrix of stock returns in the shrinkage towards singleindex model (SSIM) and the second known feature that the average correlation of stock returns is positive to estimate the covariance matrix in the shrinkage towards constant correlation model (SCCM). However, the shrinkage towards identity matrix (STIM) is a natural selection for a generic target that does not have any benefit from application specific knowledge. The STIM method tries to answer the question that whether the investors could select the optimized portfolios in the absence of finance knowledge or not. 


\subsubsection{Shrinkage Intensity}

The optimal shrinkage coefficient $\left(\delta_{*}\right)$, which is a significant finding of Ledoit and Wolf, is a trade-off between the SCM and the shrinkage target matrix. The higher coefficient of shrinkage illustrates that the more impact shrinkage methods have on the estimation of the covariance matrix. Portfolio selection performance relies on the calculation of the covariance matrix, but the projected covariance matrix is influenced by the shrinkage coefficient, and so we can assume that the essence of shrinkage methods is to find an optimal shrinkage coefficient, which is between 0 and 1 . Additionally, if the coefficient of shrinkage is low or close to 0 , it indicates that there are not many flaws in the calculation of SCM. Conversely, if the shrinkage coefficient is high and close to 1 the errors are higher.

The shrinkage coefficient is measured by the function Quadratic Loss: $\mathrm{L}(\alpha)=\left\|\delta \mathrm{F}+(1-\delta) \mathrm{S}-\sum\right\|^{2}$ In which $\mathrm{F}$ denotes the shrinkage target matrix, $\mathrm{S}$ is the sample covariance matrix and $\sum$ is the true covariance matrix. The shrinkage coefficient is an optimal value when $L(\alpha)$ is minimum.

\subsection{Portfolio Optimization}

There are various methods of choosing an optimum portfolio and one of the standard approaches of modern portfolio theory is to find a global minimum variance portfolio (GMVP) with a $\mathrm{N}$ asset universe and stock weights $w=\left(w_{1} ; w_{2} ; \ldots . ; w_{N}\right)$

The total of the stock weight will be equivalent to one $\left(\sum_{i=1}^{N} w_{i}=1\right)$, with the terms of $w_{i}>0$ implies that there is no short selling. The problem of portfolio selection is describes as:

$$
\begin{gathered}
\underbrace{\min }_{w} w^{T} \sum w \\
\text { s.t. } \quad w^{T} 1=1 \\
w_{i}>0 \quad \forall i=1, N
\end{gathered}
$$

In which: 1 denotes a vector of ones, and $\Sigma$ is the covariance matrix of $\mathrm{N}$ stocks. The theoretical approach to the problem (1) is feasible:

$$
w^{*}=\Sigma^{-1} 1\left(1^{T} \Sigma^{-1} 1\right)^{-1}
$$

The solution (2) corresponds to the inverse of the covariance matrix, which is typically calculated from the sample covariance matrix. Nonetheless, this method is also troublesome since the sample covariance is usually illconditioned and cannot even be invertible, particularly in high-dimensional portfolios. Consequently, the shrinkage estimators should be used to modify the covariance matrix parameter in the equation (1), resulting in a better solution in the equation (2).

\section{Data and Methodology}

\subsection{Input Data}

The weekly stock price is collected to input in the optimization procedure. The weekly returns are determined using all stocks involved that had been adjusted dividends and changes in the capital by stock splits. The observation sample data set $\mathrm{D}(\mathrm{t})$ then was divided into two parts $\mathrm{W}(\mathrm{t})$ and $\mathrm{V}(\mathrm{t})$. Where $\mathrm{W}(\mathrm{t})$ is known to be the initialization step for estimating the covariance matrix and initializing the first portfolio. This period is named in-the-sample. $\mathrm{V}(\mathrm{t})$ shall be regarded as the evaluation period used to test the efficiency of the estimation methods and called out-ofsample period.

Regarding more information, the cumulative data points observed in this analysis are $\mathrm{D}(\mathrm{t})=468$, referring to 468 weeks from January 2011 to December 2019. The initialization period $\mathrm{W}(\mathrm{t})=104$ weeks refers to the two-year duration from January 2011 to January 2013. The testing period $\mathrm{V}(\mathrm{t})=364$ weeks is the remaining data set from January 2013 to December 2019. All the companies listed on Ho Chi Minh City Stock Exchange (HOSE), but not the companies under 2 years period from Initial Public Offering (IPO), will be considered. The cumulative number of stocks picked is therefore 350 companies. The data is taken from HOSE and is denominated as VND. The VNINDEX, which is the Vietnam Stock Index, is used as a reference index for shrinking to a single-index-model.

\subsection{Back-Testing Process}

To evaluate the efficiency of covariance matrix shrinkage methods, a back-testing process is built and applied in this research from using a back-testing platform in Tran et al.(2020). Back-testing process supports authors in appraising the possibility and potential application of near future estimation, with the series of price value in portfolio. The considered back-testing process is conducted as follows:

Step 1: Dividing observations $\mathrm{D}(\mathrm{t})$ into two parts $\mathrm{W}$ and $\mathrm{V}$. Therein, $\mathrm{W}$ is considered as initial stage to estimate covariance matrix, usually call in-the-sample process and $\mathrm{V}$ is considered as testing stage of methodologies in portfolio selection, usually called out-of-sample process.In our study, based on the policies and settings of Vietnam stock market (for example, three days are required for selling or buying stocks), we choose weekly trading other than daily trading. Hence, the total observation is $\mathrm{D}(\mathrm{t})=416$, each data point equal to unit of time is week. Therein, initial stage $\mathrm{W}=104$ weeks within 2 years and testing stage $\mathrm{V}=312$ weeks. 
Step 2: Using the data in initial part $\mathrm{W}$ to estimate covariance matrix and use this matrix as input in the portfolio optimization for selecting the optimal portfolios. And then, the optimal portfolios will be tested on data point $t_{w+1}$ based on the portfolio performance criteria.

Step 3: Carrying out replacing data $t_{1}$ with data point $t_{w+1}$ in the initial part $\mathrm{W}$ to create $W_{1}$, and then continue the optimal portfolio selection process and evaluate results of the selection as in step 2 on data point $t_{w+2}$. This process is repeated during testing process $\mathrm{V}$ and end at data point $t_{v+w}$.

Step 4: Calculating and extracting the results during testing stage V. The portfolio performance criteria are applied to evaluate portfolio selection process $\mathrm{V}$ including: average return of portfolio, volatility of portfolio, portfolio turnover, maximum drawdown, winning rate and Jensen's Alpha.Moreover, transaction costs are also considered during the testing procedure of this study. Each time the portfolio status changes according to optimal results, the transaction costs are incurred. The trading cost would be assumed to be $0.3 \%$ for either total buying value or selling value of the portfolio each time. This figure is according to the real percentage applied in most stock firms on the Vietnam equity exchange.

The testing process is presented in the diagram below (see Figure 1):

\subsection{Performance Metrics}

Performance metrics are the criteria used to evaluate the efficiency of optimal portfolios. The common performance metrics are usually applied as return of portfolio, or risk of portfolio. The return of portfolio can be defined as the gain or loss of portfolio for a given period of time while the risk of portfolio is seen as the volatility of portfolio's return and can be measured by the variance of portfolio's return (Nguyen, 2019). Besides these common performance metrics, this paper considers the other criteria such as Sharpe ratio, Maximum drawdown, Portfolio turnover, Winning rate or Jensen's Alpha.

\subsubsection{Sharpe Ratio $(S R)$}

This is a metric of considering the profit over each unit of risk when an investor decides to invest in a portfolio, so the higher the ratio will be effective for investors (Sharpe, 1964). This ratio is determined as follows:

$$
\text { Sharpe ratio }\left(S R_{p}\right)=\frac{R_{p}-R_{f}}{\sigma_{p}}
$$

Where: $R_{p}$ is the average return of portfolio; $R_{f}$ is the risk-free rate for the evaluated period and $\sigma_{p}$ is the standard deviation of portfolio's returns.

\subsubsection{Maximum Drawdown (MDD)}

The maximum drawdown is also an important indicator for portfolio efficiency evaluation. This indicator reflects the portfolio's level of risk in the impoverished and complicated market situation. The maximum drawdown can be calculated as following:

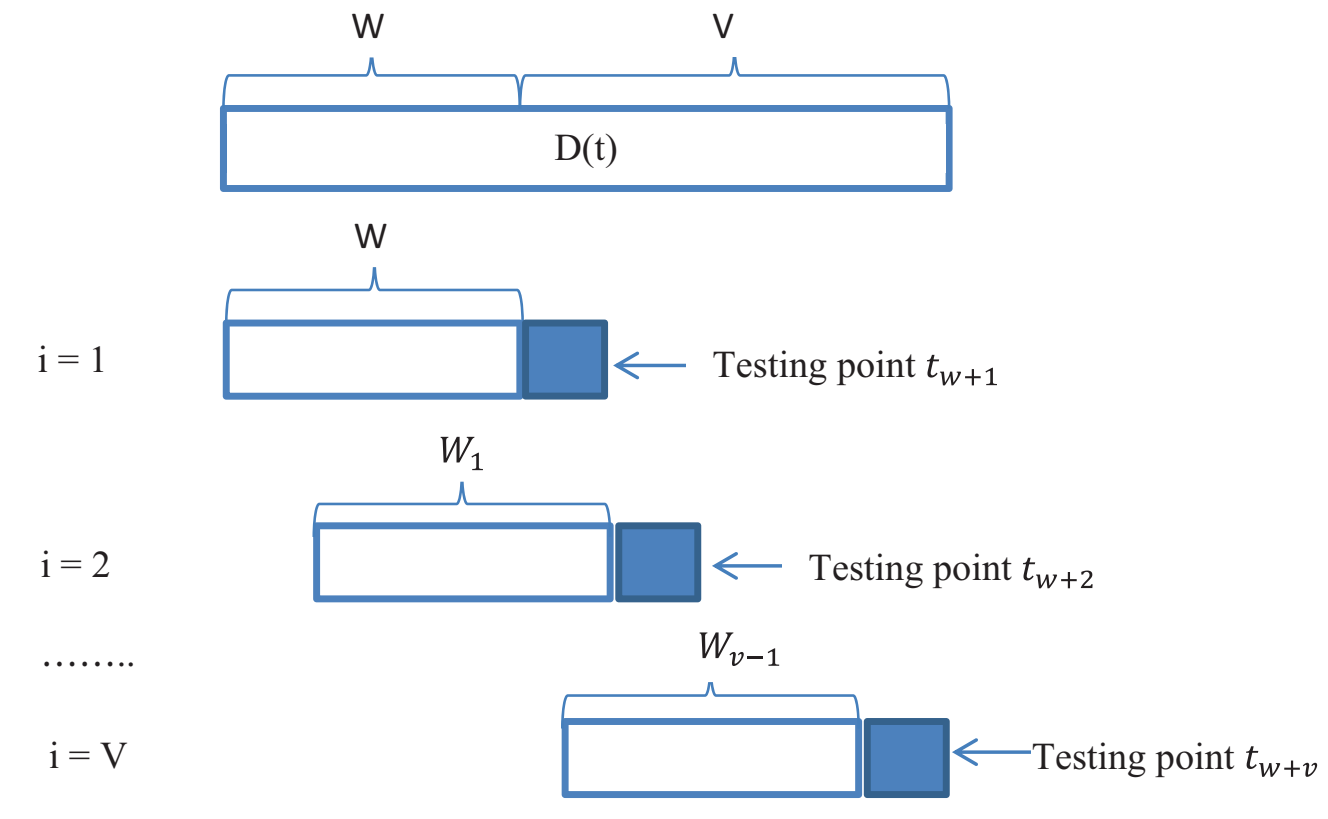

Figure 1: Back - testing procedure 
Table 1: Summary back-testing results of covariance matrix estimation techniques on out-of-sample from 1/1/2013 $31 / 12 / 2019$

\begin{tabular}{|l|c|c|c|c|c|c|c|}
\hline \multicolumn{1}{|c|}{ Methods } & $\begin{array}{c}\text { Average } \\
\text { Return }\end{array}$ & $\begin{array}{c}\text { Average } \\
\text { volatility }\end{array}$ & SR & $\begin{array}{c}\text { Daily } \\
\text { Turnover }\end{array}$ & MDD & Winning rate & Alpha \\
\hline SCM & $10.28 \%$ & $8.97 \%$ & 0.68 & $7.04 \%$ & $(23.68 \%)$ & $55.39 \%$ & $4.19 \%$ \\
\hline SSIM & $16.18 \%$ & $6.8 \%$ & 1.59 & $3.45 \%$ & $(7.87 \%)$ & $57.34 \%$ & $9.4 \%$ \\
\hline SCCM & $18.82 \%$ & $7.49 \%$ & 1.72 & $2.64 \%$ & $(8.2 \%)$ & $57.6 \%$ & $11.36 \%$ \\
\hline STIM & $16.5 \%$ & $6.5 \%$ & 1.71 & $2.26 \%$ & $(8.44 \%)$ & $58.08 \%$ & $9.59 \%$ \\
\hline
\end{tabular}

$$
M D D_{i}=\underbrace{\operatorname{argmax}}_{i, t^{*} \varepsilon(0, T)}[\underbrace{\operatorname{argmax}}_{i, t=0(0, T)}\left(\frac{V_{i, t}-V_{i, t^{*}}}{V_{i, t}}\right)]
$$

In which: $V_{i, t}$ is the portfolio value at time $\mathrm{t}$, which is optimized according to strategy i

Clearly, a lower MDD will attract the investors because it shows that the investment strategy is less risky.

\subsubsection{Portfolio Turnover}

This indicator shows the stability of the portfolio at a time when the portfolio is changing its status according to an optimal strategy. Therefore, the investors will prefer a lower turnover, because this shows that the liquidity risks will reduce and the transaction costs are also going lower. The portfolio turnover of a strategy $i$ is defined as follows:

$$
P T_{i}=\frac{1}{T} \sum_{i=1}^{T} \sum_{j=1}^{T}\left(\left|w_{i, j . t+1}-w_{i, j . t}\right|\right)
$$

In which: $\mathrm{T}$ is the number of times of portfolio change, $w_{i, j, t+1}$ is the weight of asset $\mathrm{j}$ optimized in line with strategy $\mathrm{i}$ time $\mathrm{t}+1$.

\subsubsection{Winning Rate}

The winning rate indicates that how many trades the investors win out of all their trades. The portfolios have high winning rate does not mean that they will guarantee profitability for the investors, but they can increase the winning probability of the investment. Therefore, the higher win rate will be better for portfolios.

\subsubsection{Jensen's Alpha}

This metric is a measure of the portfolio's superior return to the theoretical expected return (Le, 2018). The theoretical expected return is calculated by Capital Asset Pricing Model
(CAPM), relies on the Beta coefficient and the average market return. The metric is also generally known as the Jensen's alpha and is identified as following:

$$
\alpha=R_{p}-\left[R_{f}+\beta\left(R_{m}-R_{f}\right)\right]
$$

Where: $R_{p}$ is the average return of portfolio, $R_{f}$ is the risk free rate, $\beta$ is beta coefficient of portfolio and often estimated by Ordinary Least Square (OLS) regression (Le et al. 2018) and $R_{m}$ is the average market return.

\section{Empirical Results}

Based on back-testing results of covariance matrix estimation techniques from January 1, 2013 to December 31, 2019, we can see that the shrinkage methods give results that are completely superior to the traditional SCM method. Moreover, among the three shrinkage methods used in this study, the SCCM and STIM methods are far superior to the SSIM method on most performance metrics, but not easy to decide whether SCCM or STIM method will outperform in choosing the optimal portfolio in Vietnam stock market. Specifically, the average return of shrinkage methods in the testing period is about $17.2 \%$, much higher than the traditional SCM method, only about $10.28 \%$. In particular, the SCCM method has the highest average return of $18.82 \%$ compared to the SSIM method is $16.18 \%$ and the STIM method is $16.5 \%$ (see Table 1).

The back-testing results also showed that the average risk level of the portfolio by shrinkage methods also showed a low result of only $6.93 \%$ compared with the risk level of traditional SCM method, about $8.97 \%$ (see Figure 2). In particular, the average volatility level of the STIM method is the lowest corresponding to $6.5 \%$ compared to the volatility level of SCCM method is $7.49 \%$ and SSIM is $6.8 \%$. The Sharpe ratio of shrinkage methods is also much higher than the traditional SCM method. In particular, the Sharpe ratio of SCCM and STIM method is approximately equivalent to about 1.7 times higher than the SSIM method with a value of 1.59 times. 

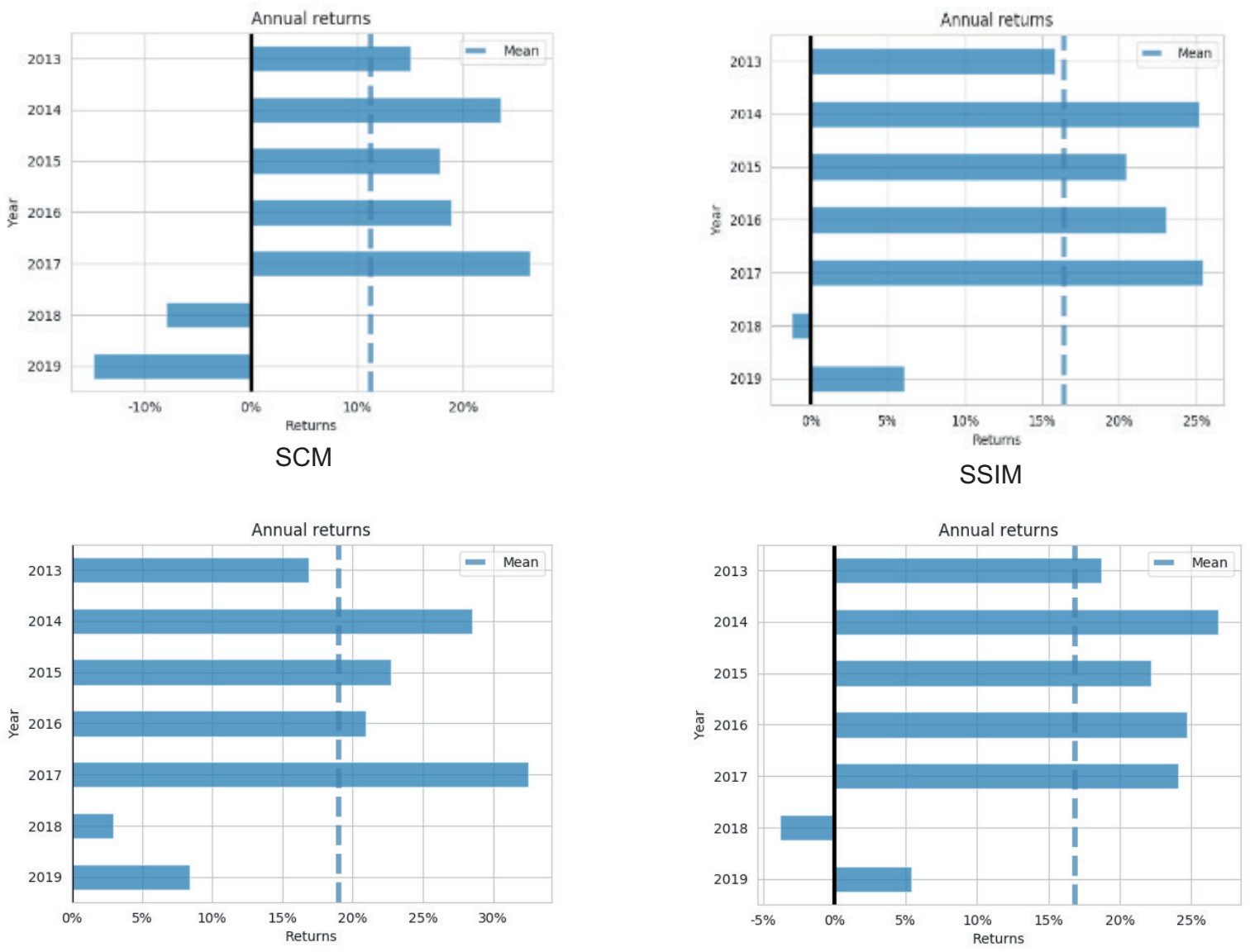

Figure 2: Back-testing results of methods' annual returns on out - of - sample from 1/1/2013 - 31/12/2019

The superiority of the shrinkage method is also shown by the Alpha coefficient during the back-testing period of 20132019 (see Figure 3). The Alpha coefficients of the shrinkage methods have a high positive value and are much higher than the SCM's Alpha. This shows that the shrinkage methods have ability to generate much higher returns than the average market return calculated by CAPM model. In particular, among the shrinkage methods, the Alpha coefficient of the SCCM reached the highest number with a value of $11.36 \%$ higher than the Alpha coefficients of the STIM and the SSIM that are valued at $9.59 \%$ and $9.4 \%$ respectively.

Moreover, considering the level of risk of portfolios in the worst-case of market conditions, the shrinkage methods offers far more secure results than the traditional SCM method. Specifically, when observing the maximum drawdown criteria of the portfolios in the period of 20132019 , we can see that the maximum loss level of the portfolios by the shrinkage methods on average is only $8.1 \%$, a percentage of very low compared to the maximum loss of traditional SCM method up to $23.68 \%$. In addition, the SSIM method has the lowest maximum loss in the back-testing period when the maximum loss is only $7.87 \%$, compared to $8.2 \%$ of the SCCM method and $8.44 \%$ of the STIM method.

Judging by the degree of change in portfolio status (Portfolio turnover), we can see that the portfolios of shrinkage methods are much more stable than the SCM method. The average daily portfolio turnover of shrinkage methods are only about $3 \%$, equivalent to about $15 \%$ of the value of the portfolio in one trading week, while the daily portfolio turnover of SCM is very high up to $7.04 \%$, corresponding to over $35 \%$ of the value of portfolio in one trading week. The portfolio turnovers of shrinkage methods are low, this means that the portfolios created by shrinkage methods will have stability and save much transaction costs. If we look closely in portfolio turnovers of three shrinkage methods, we can see that the portfolio turnover of the STIM has the lowest value which is only $2.26 \%$ /day compared to $2.64 \%$ /day of SCCM method and 3.45\%/day of SSIM method (see Figure 4). 


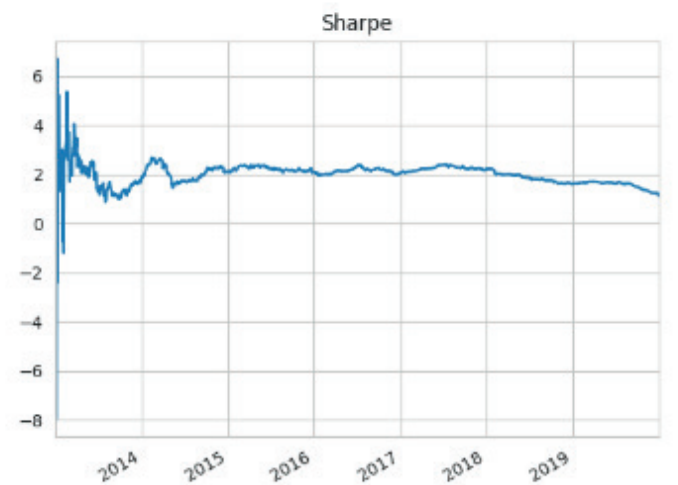

SCM

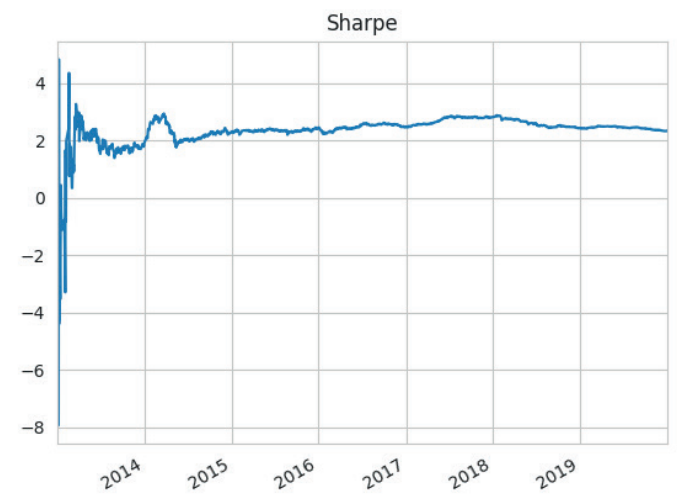

SCCM

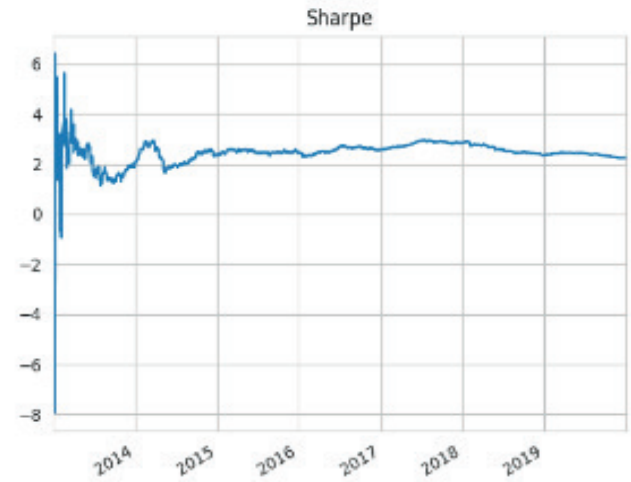

SSIM

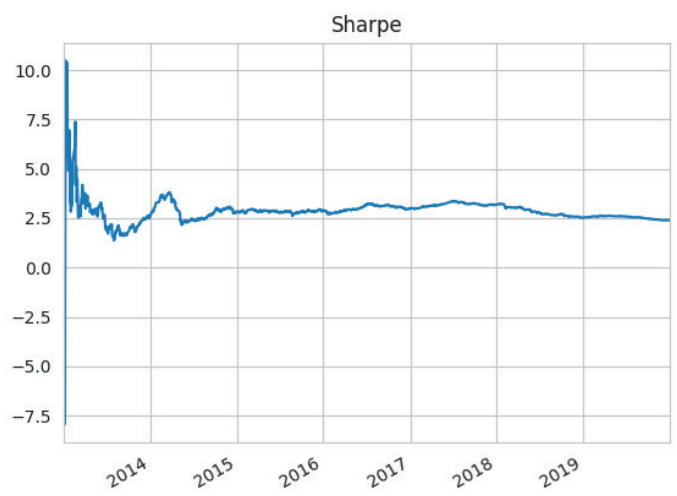

STIM

Figure 3: Back-testing results of methods' Sharpe ratios on out - of - sample from 1/1/2013 - 31/12/2019

The superiority of shrinkage method over the traditional method is also expressed through winning rate indicator. The average winning rate of shrinkage methods are up to $57.5 \%$ while the SCM method is $55.3 \%$. More specific, the winning rate of STIM is the highest value with $58.08 \%$ compared to $57.6 \%$ of SCCM and $57.3 \%$ of SSIM (see Figure 5).

When observing the optimal shrinkage coefficients of shrinkage methods, we can explain the trade-off between the SCM and the shrinkage target matrix (see Figure 6). In the period from early 2013 to the end of 2015, the shrinkage coefficients were low when fluctuating around the area from 0.05 to 0.4 . This shows that not much error occurring in estimating covariance matrix by the SCM and the estimated covariance matrix is greatly influenced by the SCM in this period. However, from 2016 to early 2018, there was a drastic change in the shrinkage coefficients, which increased quickly from 0.2 to 0.7 . This movement begins to show that there have been errors in the estimation of the SCM method and can be explained by the rapid increase in the number of stocks listed on the Vietnam stock market in the period of 2016-2018, so the estimated covariance matrix requires more intervention from the target shrinkage matrix. In the end of 2018-2019, when the errors in the SCM method showed signs of decreasing, the shrinkage coefficients had changed in the opposite direction, these coefficients decreased from the value of 0.7 to 0.4 . The movement of the shrinkage coefficient reflects the adaptation of the shrinkage methods to market changes. Moreover, although the shrinkage coefficients have the same movements among three shrinkage methods, but the movement areas of the coefficients are different. In particular, the SCCM's shrinkage coefficient move around from 0.3 to 0.7 while the SSIM's is from 0.05 to 0.55 and the STIM's 0.1 to 0.6. The higher shrinkage coefficient helps the SCCM method to be able to adjusting the estimated covariance matrix more and generating the profit more compared to the SSIM and STIM. 


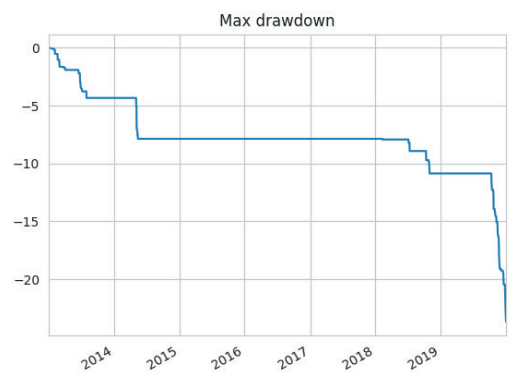

SCM

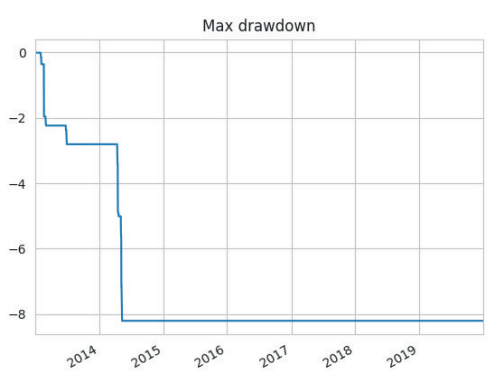

SCCM

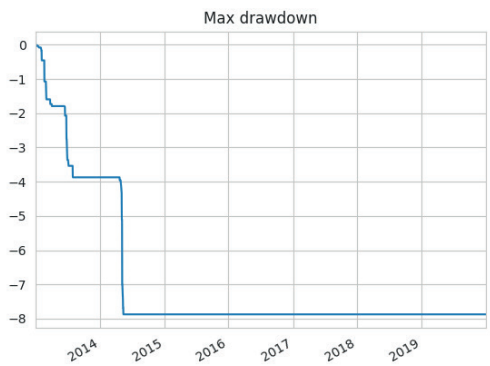

SSIM

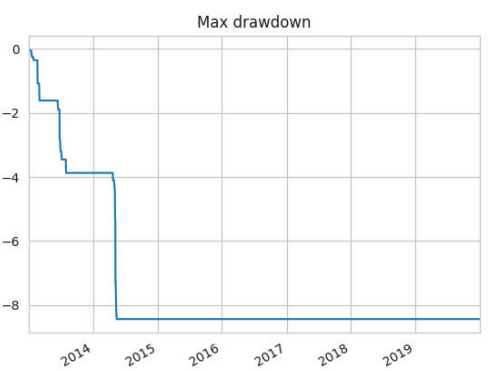

STIM

Figure 4: Back-testing results of methods' Maximum Drawdown on out - of - sample from 1/1/2013 - 31/12/2019

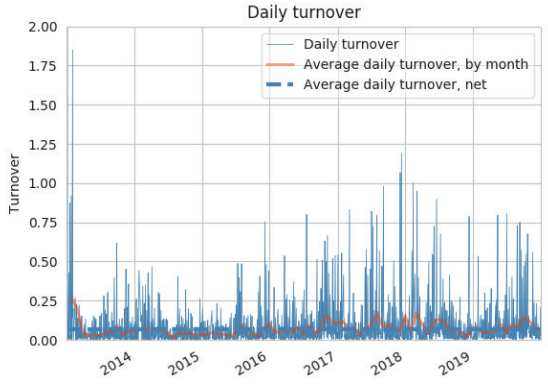

SCM

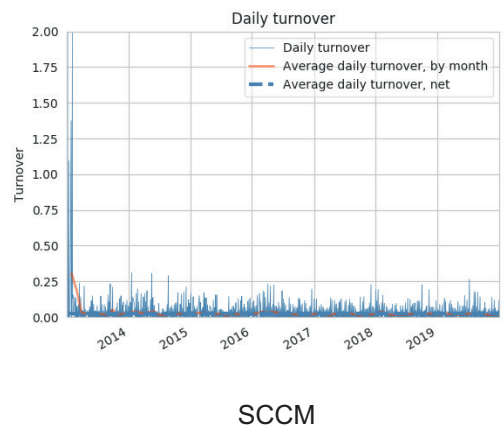

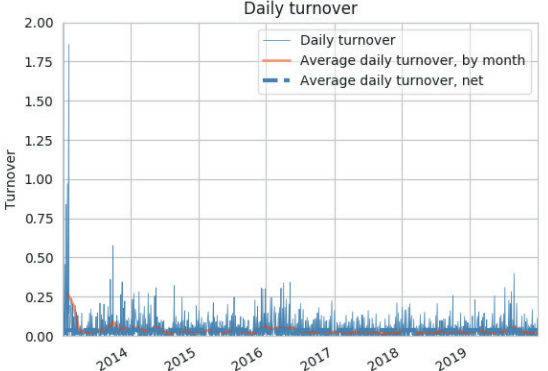

SSIM

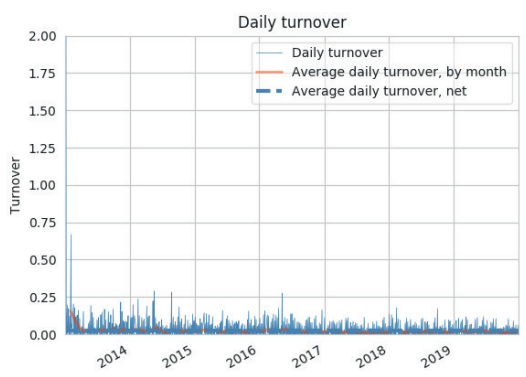

STIM

Figure 5: Back-testing results of methods' daily portfolio turnover on out - of - sample from 1/1/2013 - 31/12/2019 


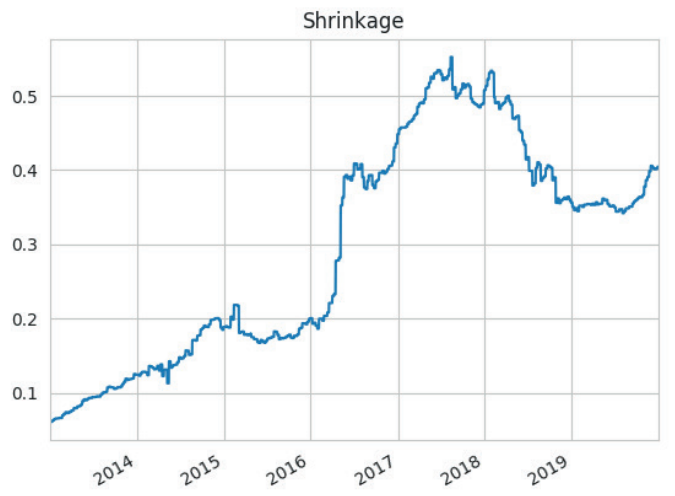

SSIM

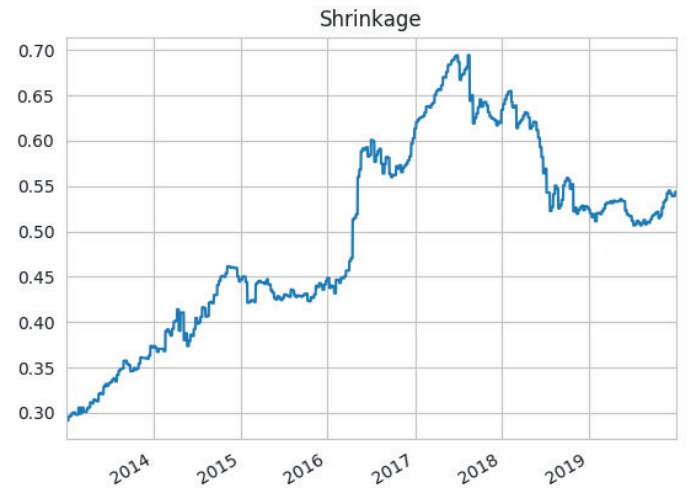

SCCM

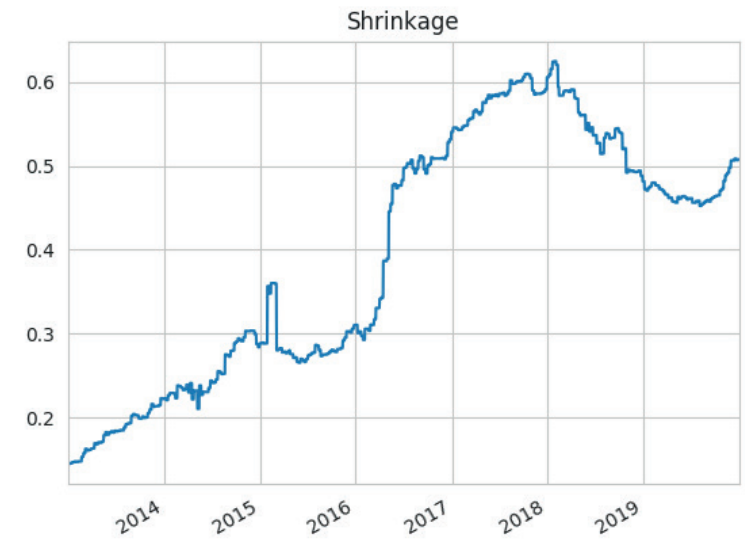

STIM

Figure 6: Back-testing results of Shrinkage intensityon out - of - sample from 1/1/2013 - 31/12/2019

\section{Conclusion and Future Works}

The empirical results show that the shrinkage of covariance matrix for portfolio optimization gives the promising results for the investors on Vietnam stock market. The shrinkage method helps the investors to produce the optimal portfolio in the sense of having higher profit with lower levels of risk compared to the portfolio of the traditional SCM method. Especially in such periods when the market faces the difficult development, the shrinkage method still generates the safe scenarios to protect our asset portfolios with the lowest maximum loss. Moreover, the portfolio turnover of shrinkage method is always kept at low magnitudes, and this makes the shrinkage portfolios save too much transaction costs and reduce the liquidity risks in trading process. In addition, the ability of shrinkage method in making profit is one again shown by the Alpha coefficient that achieves a high positive value.

Furthermore, the results of research point out which are the best shrinkage target matrices for investors in applying shrinkage methods. Based on performance metrics of portfolios, this paper shows that the shrinkage towards constant correlation (SCCM) and the shrinkage towards identity matrix (STIM) are definitely superior to the shrinkage towards single-index model (SSIM) on most of portfolio evaluation criteria. However, it is not easy to select the SCCM or STIM, which is the best one. The SCCM method has many advantages in creating highly profitable portfolios, but the STIM method is capable 
of creating safer portfolios. Besides, the other portfolio performance metrics of these two shrinkage methods are also quite similar. In the future, we will consider adapting other shrinkage target matrices, beside the ones used in this paper, to shrink the covariance matrix for portfolio optimization.

\section{References}

Bodnar, T., Okhrin, Y., \& Parolya, N. (2016). Optimal shrinkagebased portfolio selection in high dimensions. Retrieved July 14, 2019 from:https://arxiv.org/pdf/1611.01958.pdf.

Candelon, B., Hurlin, C., \& Tokpavi, S. (2012). Sampling error and double shrinkage estimation of minimum variance portfolios. Journal of Empirical Finance, 19(4), 511-527.

DeMiguel, V., Martin-Utrera, A., \&Nogales, J. F. (2013). Size matters: Optimal calibration of shrinkage estimators for portfolio selection. Journal of Banking \& Finance, 37(8), 3018-3034.

Iyiola, O., Munirat, Y., \& Christopher, N. (2012). The modern portfolio theory as an investment decision tool. Journal of Accounting and Taxation, 4(2), 19-28.

Le, T. P.(2018). Jensen's alpha estimation models in Capital Asset Pricing Model. Journal of Asian Finance, Economics and Business, 5(3), 19-29. http://doi.org/10.13106/jafeb.2018.vol5. no3.19

Le, T. P., Kim, S, K., \& Su, Y. (2018). Reexamination of estimating beta coefficient as a risk measure in CAPM. Journal of Asian Finance, Economics and Business, 5(1), 11-16. http://dx.doi. org/10.13106/jafeb.2018.vol5.no1.11

Ledoit, O., \& Wolf, M. (2003a). Improved estimation of the covariance matrix of stock returns with an application to portfolio selection. Journal of Empirical Finance, 10(5), 603-621.
Ledoit, O., \& Wolf, M. (2003b). Honey, I shrunk the sample covariance matrix. Journal of Portfolio Management, 30(4), 110-119.

Ledoit, O., \& Wolf, M. (2004). A well-conditioned estimator for large-dimensional covariance matrices. Journal of Multivariate Analysis, 88(2), 365-411.

Ledoit, O., \& Wolf, M. (2017a). Nonlinear shrinkage of the covariance matrix for portfolio selection: Markowitz meets Goldilocks. Review of Financial Studies, 30(12), 4349-4388.

Ledoit, O., \& Wolf, M. (2017b). Numerical implementation of the QuEST function. Computational Statistics \& Data Analysis, 115, 199-223. https://arxiv.org/pdf/1601.05870.pdf.

Liu, X. (2014). Portfolio optimization via generalized multivariate shrinkage. Journal of Finance \& Economics, 2(2), 54-76.

Markowitz, H. (1952). Portfolio selection. Journal of Finance, 7, 77-91.

Michaud, R. O. (1989). The Markowitz optimization enigma: Is 'optimized' optimal?. Financial Analysts Journal, 45(1), 31-42.

Nguyen, T. C., \& Nguyen, H. M.(2019). Modeling stock price volatility: Empirical evidence from the Ho Chi Minh City Stock Exchange in Vietnam. Journal of Asian Finance, Economics and Business, 6(3), 19-26. https://doi.org/10.13106/jafeb.2019. vol6.no3.19

Sharpe, F. W. (1964). Capital asset prices: A theory of market equilibrium under conditions of risk. The Journal of finance, 19(3), 425-442.

Tran,T., Nguyen, N., Nguyen, T., \& Mai, A. (2020). Voting shrinkage algorithm for Covariance Matrix Estimation and its application to portfolio selection. RIVF International Conference on Computing and Communication Technologies(pp.1-6).Ho Chi Minh City, Vietnam, October 14-15. IEEE Publishing. Retrieved July 15, 2020 from:https://ieeexplore.ieee.org/ document/9140764. 